\title{
FUNDAMENTAL CLASSES NOT REPRESENTABLE BY PRODUCTS
}

\author{
D. KOTSCHICK AND C. LÖH
}

\begin{abstract}
We prove that rationally essential manifolds with suitably large fundamental groups do not admit any maps of non-zero degree from products of closed manifolds of positive dimension. Particular examples include all manifolds of non-positive sectional curvature of rank one and all irreducible locally symmetric spaces of non-compact type. For closed manifolds from certain classes, say non-positively curved ones, or certain surface bundles over surfaces, we show that they do admit maps of non-zero degree from non-trivial products if and only if they are virtually diffeomorphic to products.
\end{abstract}

\section{INTRODUCTION}

The existence of a continuous map $M \longrightarrow N$ of non-zero degree defines an interesting transitive relation, denoted $M \geq N$, on the homotopy types of closed oriented manifolds $[29,14,7]$. In every dimension, homotopy spheres represent an absolutely minimal element for this relation. In dimension two, the relation coincides with the order given by the genus, and substantial information is now known in dimension three as well [34].

In general, if $M$ dominates $N$, then $M$ is at least as complicated as $N$. For example, $M \geq N$ implies that the Betti numbers of $M$ are at least as large as those of $N$ and that the fundamental group of $M$ surjects onto a finite index subgroup of the fundamental group of $N$. However, these necessary conditions are in general very far from being sufficient, and the relation $\geq$ is poorly understood in higher dimensions. Nevertheless, interesting results about it have been obtained for two different kinds of targets $N$ : either $N$ is assumed to be highly connected, or $N$ is assumed to be negatively curved, or at least to have a large universal covering in a suitable sense. In the highly connected case the methods of algebraic topology have been successfully applied to the

Date: June 9, 2018; (C) D. Kotschick and C. Löh 2008;

MSC 2000 classification: primary 53C23, secondary 20F34, 20F67, 57N65.

The first author is a member of the DFG priority program in Global Differential Geometry. The second author would like to thank Wolfgang Lück for clarifying discussions. 
study of the domination relation in the work of Duan and Wang [8, 9]. At the opposite end of the spectrum, for manifolds with large universal coverings, interesting results have been obtained via more geometric methods. These include Gromov's theory of bounded cohomology [14, 20], most notably the concept of simplicial volume, and the theory of harmonic maps, as applied to the domination question by Siu, Sampson, Carlson-Toledo and others, cf. [7, 1] and the literature quoted there.

In this paper we prove that certain manifolds cannot be dominated by any non-trivial product of closed manifolds. One of our motivations stems from Gromov's discussion of functorial semi-norms on homology [16, Chapter $5 G_{+}$], where the issue of representing even degree homology classes by products of surfaces is raised. Gromov suggested that many interesting homology classes should not be representable by products (of surfaces) and singled out the fundamental classes of irreducible locally symmetric spaces as specific candidates. As a special case of our results, we confirm Gromov's suggestion in the following general form: if $P$ is any non-trivial product of closed manifolds and $N$ is a closed irreducible locally symmetric space of non-compact type, then $P \nsupseteq N$; see Corollary 4.2. Another motivation for results of the type $P \nsupseteq N$ comes from the study of diffeomorphism groups, where the special case $\left(M \times S^{1}\right) \nsupseteq N$ occurs [24].

Our methods, while inspired by differential geometry and by Gromov's theory of the simplicial volume [14], are, for the most part, elementary. We combine basic homotopy theory with the discussion of certain purely algebraic properties of fundamental groups. More specifically, we translate domination by products on the level of manifolds into properties of the corresponding fundamental groups. As the images of the fundamental groups of the factors commute in the fundamental group of the target and generate a subgroup of finite index, domination by a product forces the fundamental group of the target to have a certain amount of commutativity. This alone is often enough to prove $P \nsupseteq N$.

For the formulation of our results it is convenient to use the following terminology due to Gromov [14], compare also [17].

Definition 1.1. A closed, oriented, connected $n$-manifold $N$ is called essential if $H_{n}\left(c_{N}\right)([N]) \neq 0 \in H_{n}\left(B \pi_{1}(N) ; \mathbb{Z}\right)$, where $c_{N}: N \longrightarrow B \pi_{1}(N)$ classifies the universal covering of $N$. It is rationally essential if $H_{n}\left(c_{N}\right)([N]) \neq 0$ in $H_{n}\left(B \pi_{1}(N) ; \mathbb{Q}\right)$.

Sufficient conditions to ensure essentialness are: asphericity (obviously), non-zero simplicial volume [14], enlargeability [17, 18], or the non-vanishing of certain asymptotic invariants, like the minimal volume entropy or the spherical 
volume [6]. All these properties, except possibly the last one, actually ensure rational essentialness.

We will show that domination of a manifold by a product implies that its fundamental group contains many elements with large centralisers. A simple illustration of this phenomenon is the following result proved in Section 2.

Proposition 1.2. Let $N$ be a closed, oriented, connected rationally essential manifold whose fundamental group has finite centre. For no closed, oriented, connected manifold $M$ is there a map $M \times S^{1} \longrightarrow N$ of degree 1 .

Results in a similar spirit arise in the study of diffeomorphism groups [24].

In order to exclude more general products, we require that the fundamental group of the target manifold cannot be dominated by a non-trivial product in the following sense:

Definition 1.3. An infinite group $\Gamma$ is not presentable by a product if, for every homomorphism $\varphi: \Gamma_{1} \times \Gamma_{2} \longrightarrow \Gamma$ onto a subgroup of finite index, one of the factors $\Gamma_{i}$ has finite image $\varphi\left(\Gamma_{i}\right) \subset \Gamma$.

Using Definition 1.3, which will be analysed in detail in Section 3, we prove the following topological result in Section 2:

Theorem 1.4. If $N$ is a closed, oriented, connected rationally essential manifold whose fundamental group is not presentable by a product, then $P \nsupseteq N$ for any non-trivial product $P$ of closed, oriented, connected manifolds.

This is complemented by an algebraic result, proved in Section 3, providing examples of groups not presentable by products:

Theorem 1.5. The following groups are not presentable by products:

$(\mathrm{H})$ hyperbolic groups that are not virtually cyclic,

(N-P) fundamental groups of closed Riemannian manifolds of non-positive sectional curvature of rank one and of dimension $\geq 2$,

(MCG) mapping class groups of closed oriented surfaces of genus $\geq 1$.

The rank occurring in statement (N-P) can be taken to be either the geometric rank of the Riemannian metric [2], or the rank of the fundamental group [3]. It is a result of Ballmann-Eberlein [3] that the two agree. In Section 7, we discuss the rôle of this rank in our context.

Of course, as the groups in Theorem 1.5 are of geometric origin, the proof uses information gleaned from geometry. The case of fundamental groups of strictly negatively curved manifolds is contained as a special case in both $(\mathrm{H})$ and (N-P). For these groups it is an elementary application of Preissmann's theorem to show that they are not presentable by products. Our proofs of the 
cases $(\mathrm{H})$ and $(\mathrm{MCG})$ follow the same line of argument, using the fact that most elements of those groups have small centralisers.

Theorem 1.5, particularly statement $(\mathrm{H})$, shows that our methods are well suited to the study of targets that have some sort of negative curvature property. This is also true for the applications of the simplicial volume and of harmonic maps mentioned earlier. However, in contrast with those techniques, our methods also apply to manifolds and groups that are not non-positively curved at all. For instance, the mapping class groups of surfaces of genus $\geq 2$ occurring in Theorem 1.5 are not hyperbolic because they contain Abelian subgroups of large rank. In fact, they do not even have any semi-simple actions by isometries on $\mathrm{CAT}(0)$-spaces $[23,4]$.

In the second half of this paper we generalise our results following the philosophy of extending from the hyperbolic to a suitable semi-hyperbolic situation. In Section 4 the classical case of Riemannian manifolds of non-positive sectional curvature is considered. For such manifolds we prove that being dominated by a product is equivalent to being virtually diffeomorphic to a product. The result about closed irreducible locally symmetric spaces of non-compact type predicted by Gromov is a special case of this more general theorem. Similarly, in Section 5 we prove that the total spaces of certain fibrations are dominated by products if and only if they are virtually trivial.

In Section 6 we show that our assumptions on the fundamental group can be relaxed at the expense of making a stronger assumption than rational essentialness on the manifold we are dealing with. Finally in Section 7 we discuss the domination relation more generally, not restricting to product domains, and also considering targets with finite fundamental groups.

\section{The topological ARGUMENT}

In this section we investigate the relation between presentability by products on the level of fundamental groups and domination of rationally essential manifolds by non-trivial products. In particular, we prove Proposition 1.2 and Theorem 1.4.

Throughout this section, we consider the following situation. We suppose that $N$ is a closed, oriented, connected $n$-manifold, and $f: X_{1} \times X_{2} \longrightarrow N$ is a map of degree $d \neq 0$ from a non-trivial product of closed, oriented, connected manifolds $X_{i}$. We choose base points $x_{i} \in X_{i}$ and $f\left(x_{1}, x_{2}\right) \in N$. All fundamental groups are taken with respect to these base points. We write

$$
\begin{aligned}
& f_{1}:=\left.f\right|_{X_{1} \times\left\{x_{2}\right\}}: X_{1} \times\left\{x_{2}\right\} \longrightarrow N, \\
& f_{2}:=\left.f\right|_{\left\{x_{1}\right\} \times X_{2}}:\left\{x_{1}\right\} \times X_{2} \longrightarrow N,
\end{aligned}
$$




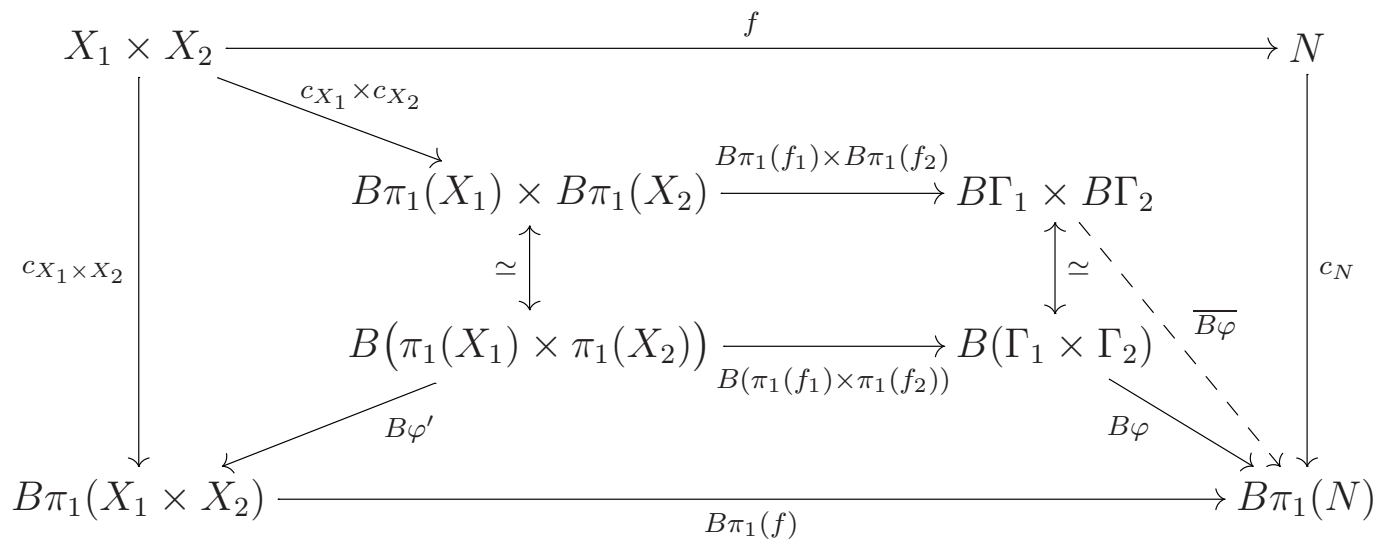

FiguRE 1. Naturality of classifying maps

and set $\Gamma_{i}:=\operatorname{im}\left(\pi_{1}\left(f_{i}\right)\right) \subset \pi_{1}(N)$. Finally, let $\Gamma:=\operatorname{im}\left(\pi_{1}(f)\right) \subset \pi_{1}(N)$.

Lemma 2.1. The subgroup $\Gamma$ has finite index in $\pi_{1}(N)$ and multiplication in $\pi_{1}(N)$ defines a surjective homomorphism $\varphi: \Gamma_{1} \times \Gamma_{2} \longrightarrow \Gamma$.

Proof. As $f$ has non-zero degree, covering space theory shows that the image $\Gamma=\operatorname{im}\left(\pi_{1}(f)\right)$ has finite index in $\pi_{1}(N)$. The second statement follows because $\Gamma_{1} \cup \Gamma_{2}$ generates $\Gamma$, and the $\Gamma_{i}$ commute with each other.

Proposition 2.2. The diagram in Figure 1 is commutative. In particular, there are homology classes $\alpha_{1} \in H_{\operatorname{dim} X_{1}}\left(B \Gamma_{1} ; \mathbb{Q}\right)$ and $\alpha_{2} \in H_{\operatorname{dim} X_{2}}\left(B \Gamma_{2} ; \mathbb{Q}\right)$ satisfying

$$
H_{n}\left(c_{N}\right)(d \cdot[N])=H_{n}(\overline{B \varphi})\left(\alpha_{1} \times \alpha_{2}\right)
$$

Proof. We first explain the notation occurring in Figure 1. For a connected manifold $M$, we write $c_{M}: M \longrightarrow B \pi_{1}(M)$ for the classifying map of the universal covering. Recall that every homomorphism $\psi: K^{\prime} \longrightarrow K$ of groups yields a continuous map $B \psi: B K^{\prime} \longrightarrow B K$ that induces the given homomorphism $\psi$ on the level of fundamental groups; moreover, $B \psi$ is characterised uniquely up to homotopy by this property.

The vertical homotopy equivalences in the centre of the diagram are induced by the projections/inclusions on the level of groups. The map $B \varphi^{\prime}$ is induced by the canonical isomorphism $\varphi^{\prime}: \pi_{1}\left(X_{1}\right) \times \pi_{1}\left(X_{2}\right) \longrightarrow \pi_{1}\left(X_{1} \times X_{2}\right)$ given by the inclusions. Finally, $B \varphi$ is the map induced by the homomorphism $\varphi$ in Lemma 2.1. 
It is a routine matter to verify that the diagram in Figure 1 is commutative up to homotopy. By the homotopy invariance of homology, the corresponding diagram in homology is also commutative.

In the following, we abbreviate the composition of $B \varphi$ with the homotopy equivalence $B \Gamma_{1} \times B \Gamma_{2} \longrightarrow B\left(\Gamma_{1} \times \Gamma_{2}\right)$ by $\overline{B \varphi}: B \Gamma_{1} \times B \Gamma_{2} \longrightarrow B \pi_{1}(N)$. Using the naturality of the homological cross-product, we obtain the following relation in homology:

$$
\begin{aligned}
H_{n}\left(c_{N}\right)(d[N]) & =H_{n}\left(c_{N}\right) \circ H_{n}(f)\left(\left[X_{1} \times X_{2}\right]\right) \\
& =H_{n}\left(c_{N}\right) \circ H_{n}(f)\left(\left[X_{1}\right] \times\left[X_{2}\right]\right) \\
& =H_{n}(\overline{B \varphi}) \circ H_{n}\left(\left(B \pi_{1}\left(f_{1}\right) \times B \pi_{1}\left(f_{2}\right)\right) \circ\left(c_{X_{1}} \times c_{X_{2}}\right)\right)\left(\left[X_{1}\right] \times\left[X_{2}\right]\right) \\
& =H_{n}(\overline{B \varphi})\left(\alpha_{1} \times \alpha_{2}\right),
\end{aligned}
$$

where we put $\alpha_{i}:=H_{\operatorname{dim} X_{i}}\left(B \pi_{1}\left(f_{i}\right) \circ c_{X_{i}}\right)\left(\left[X_{i}\right]\right) \in H_{\operatorname{dim} X_{i}}\left(B \Gamma_{i} ; \mathbb{Q}\right)$.

The proofs of the topological results stated in the introduction are now completely straightforward.

Proof of Proposition 1.2. If $f: M \times S^{1} \longrightarrow N$ has degree one, then $B \pi_{1}(f)$ is surjective, and so it sends the fundamental group of the circle to the centre $C\left(\pi_{1}(N)\right)$ of $\pi_{1}(N)$. As this centre is assumed to be finite, its classifying space has trivial rational homology and we conclude

$$
H_{1}\left(B \pi_{1}\left(\left.f\right|_{S^{1}}\right) \circ c_{S^{1}}\right)\left(\left[S^{1}\right]\right)=0 \in H_{1}\left(B C\left(\pi_{1}(N)\right) ; \mathbb{Q}\right) .
$$

Now Proposition 2.2 (with $d=1$ ) shows that $H_{n}\left(c_{N}\right)([N])$ vanishes, contradicting the rational essentialness of $N$.

Proof of Theorem 1.4. Let $N$ be a closed, oriented, connected manifold, and assume that there exists a map $f: X_{1} \times X_{2} \longrightarrow N$ of non-zero degree. If $N$ is rationally essential, then the homology classes $\alpha_{i}$ in Proposition 2.2 are both non-trivial and in positive degrees. Therefore the groups $\Gamma_{i}$ are both infinite. Now Lemma 2.1 shows that $\pi_{1}(N)$ is presented by the product $\Gamma_{1} \times \Gamma_{2}$.

\section{Groups not presentable by products}

In this section we discuss groups not presentable by products. In particular we prove Theorem 1.5, and we generalise the result to certain groups arising as extensions. 
Preliminaries. Consider a homomorphism $\varphi: \Gamma_{1} \times \Gamma_{2} \longrightarrow \Gamma$. Without loss of generality we can replace each $\Gamma_{i}$ by its image in $\Gamma$ under the restriction of $\varphi$, so that we may assume the factors $\Gamma_{i}$ to be subgroups of $\Gamma$ and $\varphi$ to be multiplication in $\Gamma$.

Lemma 3.1. If $\Gamma$ is not presentable by a product, then every finite index subgroup has finite centre.

Proof. If $\bar{\Gamma} \subset \Gamma$ is a subgroup of finite index with infinite centre $C(\bar{\Gamma})$, then the multiplication map $\bar{\Gamma} \times C(\bar{\Gamma}) \longrightarrow \Gamma$ shows that $\Gamma$ is presentable by a product.

The following is a kind of converse to this observation:

Proposition 3.2. If every subgroup of finite index in $\Gamma$ has trivial centre, then $\Gamma$ is irreducible if and only if it is not presentable by a product.

Before the proof of this proposition, we need another lemma.

Lemma 3.3. Let $\Gamma_{1}, \Gamma_{2} \subset \Gamma$ be commuting subgroups with the property that $\Gamma_{1} \cup \Gamma_{2}$ generates $\Gamma$. Then the multiplication homomorphism $\varphi: \Gamma_{1} \times \Gamma_{2} \longrightarrow \Gamma$ is well-defined and surjective and the following statements hold:

(1) the intersection $\Gamma_{1} \cap \Gamma_{2} \subset \Gamma$ is a subgroup of the centre of $\Gamma$, and

(2) the kernel of $\varphi$ is isomorphic to the Abelian group $\Gamma_{1} \cap \Gamma_{2}$.

Proof. The first statement is true because the $\Gamma_{i}$ generate $\Gamma$ and commute with each other. So, if an element of $\Gamma$ is in both $\Gamma_{i}$, then it commutes with all generators. It follows in particular that $\Gamma_{1} \cap \Gamma_{2}$ is Abelian.

For the second statement observe that $\left(g_{1}, g_{2}\right) \in \Gamma_{1} \times \Gamma_{2}$ maps to the neutral element of $\Gamma$ if and only if $g_{1}=g_{2}^{-1}$ in $\Gamma$. But $g_{1} \in \Gamma_{1}, g_{2}^{-1} \in \Gamma_{2}$, which implies $g_{1}, g_{2} \in \Gamma_{1} \cap \Gamma_{2}$. Thus, the anti-diagonal $\Gamma_{1} \cap \Gamma_{2} \longrightarrow \Gamma, g \longmapsto\left(g, g^{-1}\right)$ is an isomorphism onto the kernel of $\varphi$.

Proof of Proposition 3.2. Suppose that such a $\Gamma$ is reducible in the sense that it has a finite index subgroup that is a direct product of infinite groups. Then obviously $\Gamma$ is presentable by a product.

Conversely, assume that $\varphi: \Gamma_{1} \times \Gamma_{2} \longrightarrow \Gamma$ is surjective onto a subgroup $\bar{\Gamma} \subset \Gamma$ of finite index, and that $\varphi\left(\Gamma_{i}\right)$ is infinite for both $i$. Then Lemma 3.3 applied to the subgroups $\varphi\left(\Gamma_{1}\right)$ and $\varphi\left(\Gamma_{2}\right)$ of $\bar{\Gamma}$ shows that $\Gamma$ must be reducible, because we assumed that all finite index subgroups have trivial centre.

Sometimes it is convenient to replace a given group by a subgroup of finite index. This transition does not affect presentability by products by the following straightforward observation: 
Lemma 3.4. Let $\Gamma$ be a group. A finite index subgroup $\bar{\Gamma} \subset \Gamma$ is presentable by a product if and only if $\Gamma$ is.

Hyperbolic groups. We now show that most hyperbolic groups are not presentable by products, which is case $(\mathrm{H})$ of Theorem 1.5. The following lemma is probably well known, but we did not find it explicitly in the literature.

Lemma 3.5. Let $\Gamma$ be a hyperbolic group that is not virtually cyclic. Then the centre $C(\Gamma)$ is finite.

Proof. Suppose the centre $C(\Gamma)$ contains a non-trivial element $g$. By definition, the centraliser $C_{\Gamma}(g)$ of such a $g$ is the whole group $\Gamma$. If $g$ had infinite order, then $C_{\Gamma}(g)=\Gamma$ would be virtually cyclic [4, Corollary 3.10 on p. 462], contradicting the hypothesis on $\Gamma$. Hence, all elements of $C(\Gamma)$ have finite order.

As $\Gamma$ is not virtually cyclic, $\Gamma$ must be infinite; thus, $\Gamma$ contains an element $g$ of infinite order [4, Proposition 2.22 on p. 458]. The torsion group $C(\Gamma)$ is a subgroup of the centraliser $C_{\Gamma}(g)$, which is virtually cyclic. So $C(\Gamma)$ is finite.

Proposition 3.6. A hyperbolic group that is not virtually cyclic is not presentable by a product.

Proof. Let $\Gamma$ be a hyperbolic group that is not virtually cyclic and suppose that $\varphi: \Gamma_{1} \times \Gamma_{2} \longrightarrow \Gamma$ is a homomorphism onto a finite index subgroup. Because finite index subgroups of hyperbolic groups are hyperbolic, we may assume that $\varphi$ is surjective. Furthermore, we may assume without loss of generality that the $\Gamma_{i}$ are subgroups of $\Gamma$ and that $\varphi$ is just the multiplication map.

By Lemma 3.3 the intersection $\Gamma_{1} \cap \Gamma_{2}$ is contained in the centre of $\Gamma$, and is therefore finite by Lemma 3.5.

Because $\Gamma$ is not finite, it contains an element of infinite order [4, Proposition 2.22 on p. 458]. Therefore we may assume that $\Gamma_{1}$ contains an element $g_{1}$ of infinite order. The group $\Gamma_{2}$ is contained in $C_{\Gamma}\left(g_{1}\right)$ and hence is virtually cyclic [4, Corollary 3.10 on p. 462].

Now assume that $\Gamma_{2}$ is also infinite. Then $\Gamma_{2}$ contains an element $g_{2}$ of infinite order (because the group $\Gamma_{2}$ is virtually cyclic). Now $g_{1}$ and $g_{2}$ generate a copy of $\mathbb{Z} \times \mathbb{Z}$ in $\Gamma$. For if this were false, then a power of $g_{1}$ would equal a power of $g_{2}$, and these powers would be contained in the finite group $\Gamma_{1} \cap \Gamma_{2}$; this is not possible because the $g_{i}$ have infinite order. However, hyperbolic groups cannot contain $\mathbb{Z} \times \mathbb{Z}$ because centralisers of elements of infinite order are virtually cyclic. This contradiction shows that one of the $\Gamma_{i}$ must be finite. 
Fundamental groups of non-positively curved manifolds. The following proposition corresponds to the case (N-P) of Theorem 1.5.

Proposition 3.7. If $\Gamma$ is the fundamental group of a closed Riemannian manifold $N$ of non-positive sectional curvature of rank one and of dimension $\geq 2$, then $\Gamma$ is not presentable by a product.

Proof. Recall that by the result of Ballmann-Eberlein [3] the geometric rank of $N$ coincides with the algebraic rank of $\Gamma$, that this rank is additive under direct products of manifolds respectively of groups, and that it is invariant under passage to finite coverings respectively to finite index subgroups. The assumption that $N$ be of rank one therefore implies that $N$ is locally irreducible and that $\Gamma$ is irreducible (any direct factor of $\Gamma$ would be infinite because the group is torsion-free).

The irreducibility of $N$ and the assumption $\operatorname{dim} N \geq 2$ imply that $N$ has no Euclidean local de Rham factor, so that by the result of Eberlein [11, p. 210f], the centres of $\Gamma$ and of all its finite index subgroups are trivial. Now the conclusion follows from Proposition 3.2.

We will generalise this Proposition in the course of the proof of Theorem 4.1 in Section 4.

Mapping class groups. Finally we deal with the case (MCG) in Theorem 1.5. We refer the reader to Ivanov's book [22] for the relevant information on mapping class groups and their subgroups.

A mapping class is called irreducible if it does not fix any non-trivial isotopy class of a curve on the surface. The same terminology is applied to subgroups of the mapping class group. Thus a subgroup is said to be irreducible if there is no isotopy class of a curve on the surface fixed by the whole subgroup.

Proposition 3.8. Mapping class groups of closed, oriented surfaces of genus at least 1 are not presentable by products.

Proof. In genus one the mapping class group is $\mathrm{SL}_{2}(\mathbb{Z})$. This group is virtually free and hence hyperbolic. Thus we may appeal to Proposition 3.6 to cover this case.

Now consider the mapping class group of a closed surface of genus $\geq 2$. After passing to a subgroup of finite index, we may assume that we are dealing with a torsion-free group $\Gamma$ (compare Lemma 3.4). Assume that $\Gamma_{1}, \Gamma_{2} \subset \Gamma$ are non-trivial commuting subgroups for which the multiplication $\Gamma_{1} \times \Gamma_{2} \longrightarrow \Gamma$ is surjective. 
As each $\Gamma_{i}$ is infinite and normal in the irreducible group $\Gamma$, it is itself irreducible [22, Corollary 7.13]. It follows that each $\Gamma_{i}$ contains a pseudoAnosov element [22, Corollary 7.14]. Now if $g_{1} \in \Gamma_{1}$ is pseudo-Anosov, then the centraliser $C_{\Gamma}\left(g_{1}\right)$ of $g_{1}$ in $\Gamma$ is cyclic [22, Lemma 8.13] and contains $\Gamma_{2}$. Thus $\Gamma_{2}$ is cyclic. Reversing the rôles of $\Gamma_{1}$ and $\Gamma_{2}$ we see that $\Gamma_{1}$ is cyclic as well. Thus we reach the absurd conclusion that the mapping class group is virtually Abelian. This contradiction shows that the mapping class group is not presentable by a product.

Group extensions. Having completed the proof of Theorem 1.5, we now extend our results to other groups and manifolds. In this direction, we will use the following result to study fibrations in Section 5:

Proposition 3.9. Assume that the group $\Gamma$ fits into an extension of the form

$$
1 \longrightarrow K \longrightarrow \Gamma \stackrel{\pi}{\longrightarrow} Q \longrightarrow 1
$$

where both $K$ and $Q$ are torsion-free, non-trivial and not presentable by products. Then $\Gamma$ is presentable by a product if and only if it has a finite index subgroup that is a direct product of finite index subgroups of $K$ and $Q$.

Proof. One direction is clear: if $\Gamma$ has a finite index subgroup that is a direct product of non-trivial torsion-free groups, then it is presented by a product.

For the converse, let $\varphi: \Gamma_{1} \times \Gamma_{2} \longrightarrow \Gamma$ be surjective onto a finite index subgroup. After replacing $\Gamma$ by this subgroup (and suitably replacing $K$ and $Q$ by the corresponding finite index subgroups), we may assume that $\varphi$ is surjective. As before, we may also assume that the $\Gamma_{i}$ are subgroups of $\Gamma$. Assume that they are both non-trivial.

Now consider $\pi \circ \varphi: \Gamma_{1} \times \Gamma_{2} \longrightarrow Q$. This is surjective. As $Q$ is torsion-free and not presentable by a product, one of the $\Gamma_{i}$ has trivial image. Let us assume that this is $\Gamma_{1}$. Then by the exactness of (1), we have $\Gamma_{1} \subset K$.

If $\Gamma_{1}$ and $K \cap \Gamma_{2}$ are both infinite, then we have a contradiction with the assumption that $K$ is not presentable by a product. So one of these groups is finite. As $K$ is torsion-free, such a finite subgroup must be trivial. But $\Gamma_{1}$ is non-trivial by assumption, so we conclude that $K \cap \Gamma_{2}$ is trivial, and so $\pi$ maps $\Gamma_{2}$ injectively onto $Q$.

We now claim that $\varphi: \Gamma_{1} \times \Gamma_{2} \longrightarrow \Gamma$ is an isomorphism. For if $\varphi\left(g_{1}, g_{2}\right)$ is trivial, then so is $\pi\left(\varphi\left(g_{1}, g_{2}\right)\right)=\left(\pi \mid \Gamma_{2}\right)\left(g_{2}\right)$, which implies that $g_{2}$ is trivial. Hence, the triviality of $\varphi\left(g_{1}, g_{2}\right)=\varphi\left(g_{1}, e\right)$ shows that $g_{1}$ is trivial in $\Gamma_{1}$.

As an immediate application, we can extend Proposition 3.8 to mapping class groups of surfaces with a marked point. Here we consider diffeomorphisms of a surface fixing the marked point, up to isotopies fixing the point. 
Corollary 3.10. The mapping class group of a closed, oriented surface of genus $\geq 2$ with a marked point is not presentable by a product.

Proof. Let $\Gamma$ be the mapping class group of a closed, oriented surface $\Sigma$ with respect to a marked point. We have an exact sequence

$$
1 \longrightarrow \pi_{1}(\Sigma) \longrightarrow \Gamma \stackrel{\pi}{\longrightarrow} Q \longrightarrow 1,
$$

where $Q$ is the mapping class group of $\Sigma$ (without a marked point) considered in Proposition 3.8, and $\pi$ is the forgetful map. We may pull back this extension to a torsion-free finite index subgroup of $Q$, so that the assumption on the quotient in Proposition 3.9 is satisfied. Note that the kernel is the fundamental group of a closed manifold of negative curvature, and so is not presentable by a product, for example by Proposition 3.6. The conclusion follows from Proposition 3.9, because the (universal) extension (2) is non-trivial when restricted to any finite index subgroups of $\pi_{1}(\Sigma)$ and of $Q=\operatorname{Out}\left(\pi_{1}(\Sigma)\right)$.

\section{Manifolds of NON-POSitive CURVATURE}

In this section we discuss the domination of closed manifolds of non-positive sectional curvature by products. The combination of Theorems 1.4 and 1.5 shows that $P \ngtr N$ whenever $P$ is a non-trivial product of closed manifolds and $N$ is a non-positively curved closed Riemannian manifold of rank one; this includes in particular all closed Riemannian manifolds of negative sectional curvature. Most non-positively curved manifolds are of rank one, even when they have a great deal of zero curvature $[2,3,12]$, but it remains to deal with the general case. The following result shows that domination by a product is only possible for a non-positively curved manifold if it is itself virtually a product.

Theorem 4.1. Let $N$ be a closed, oriented, connected Riemannian manifold with non-positive sectional curvature and $\Gamma$ its fundamental group. If the dimension of $N$ is at least two, then the following properties are equivalent:

(1) $P \geq N$ for some non-trivial product $P$ of closed, oriented manifolds,

(2) the fundamental group $\Gamma$ is presentable by a product,

(3) some finite index subgroup of $\Gamma$ splits as a non-trivial direct product,

(4) there is a finite covering of $N$ diffeomorphic to a non-trivial product $N_{1} \times N_{2}$ of closed, oriented manifolds $N_{i}$.

Proof. Recall that by the Cartan-Hadamard theorem manifolds of non-positive curvature are aspherical, and so are classifying spaces for their fundamental groups. Therefore, (1) implies (2) by Theorem 1.4. 
Assume that (2) holds. Then there is a homomorphism $\varphi: \Gamma_{1} \times \Gamma_{2} \longrightarrow \Gamma$ that is surjective onto a finite index subgroup $\bar{\Gamma} \subset \Gamma$ such that both $\Gamma_{i}$ have infinite image. As discussed in Section 3 we may take the $\Gamma_{i}$ to be subgroups of $\Gamma$ and $\varphi$ to be multiplication. If $\bar{\Gamma}$ has trivial centre, then Lemma 3.3 shows that $\varphi$ is injective, and so $\bar{\Gamma}$ is a non-trivial direct product. If $\bar{\Gamma}$ has non-trivial centre, then the centre is infinite because $\Gamma$ is torsion-free. A result of Eberlein [10] shows that $\bar{\Gamma}$ has a finite index subgroup that splits off $C(\bar{\Gamma})$ as a direct factor. This gives a non-trivial splitting of the subgroup as a direct product, because we assumed $\operatorname{dim} N>1$, which implies that $\Gamma$ is not virtually $\mathbb{Z}$. Thus (2) implies (3).

Next, assume that (3) holds, so that there is a finite index subgroup $\bar{\Gamma}$ of $\Gamma$ that splits as a non-trivial direct product $\Gamma_{1} \times \Gamma_{2}$. If the group $\bar{\Gamma}$ has trivial centre, then the Gromoll-Wolf [13] splitting theorem gives an isometric splitting $\bar{N}=N_{1} \times N_{2}$, where $\bar{N}$ is the covering of $N$ corresponding to the subgroup $\bar{\Gamma}$, and $\pi_{1}\left(N_{i}\right)=\Gamma_{i}$. If the centre of $\bar{\Gamma}$ is non-trivial, then again by Eberlein's results [10] some finite covering splits off a torus as a direct factor. (This splitting is usually not isometric $[27,10]$.) Thus (3) implies (4).

Finally, the implication from (4) to (1) is trivial.

The implication from (1) to (4) shows that manifolds of non-positive curvature dominated by products are virtually diffeomorphic to products. As an immediate consequence of this, we confirm Gromov's suggestion [16, 5.36 on p. 303f]:

Corollary 4.2. Let $N$ be a closed locally symmetric space of non-compact type. Then $P \geq N$ for some non-trivial product $P$ if and only if $N$ is reducible in the sense that it has a finite covering isometric to a product.

Proof. Theorem 4.1 shows that $P \geq N$ is equivalent to $N$ having a finite covering diffeomorphic to a non-trivial product. In the case that $\pi_{1}(N)$ has trivial centre, the proof of Theorem 4.1 also shows that this diffeomorphic splitting is in fact isometric. Thus we only have to check that the centre of $\pi_{1}(N)$ is trivial.

For $N$ to be a locally symmetric space of non-compact type means that the universal covering $\tilde{N}$ is a globally symmetric space without compact or Euclidean factors in its de Rham decomposition. There are two ways to see that this implies the triviality of the centre of $\pi_{1}(N)$, geometrically or algebraically. Geometrically, the centre of the fundamental group of any closed manifold of non-positive curvature is contained in the Clifford subgroup, which, by a result of Eberlein [11], is of rank equal to the dimension of the Euclidean de Rham factor of $\tilde{N}$. Algebraically, for a locally symmetric space the fundamental 
group is a lattice in a semisimple Lie group with finite centre. In the case of a symmetric space with no compact or Euclidean factors the isometry group does not have any compact factors either, and standard results about lattices show that torsion-free cocompact lattices have trivial centre [32, Corollary 5.18], [35, Corollary 4.41].

Combining our results with a result of Ballmann-Eberlein [3], we have the following:

Corollary 4.3. Let $N$ be a closed, oriented, connected Riemannian manifold of non-positive sectional curvature, and $\Gamma$ its fundamental group. The four conditions in Theorem 4.1 hold for $N$, respectively for $\Gamma$, if and only if $N$, respectively $\Gamma$, is of rank $\geq 2$ and $N$ is not an irreducible locally symmetric space of non-compact type.

Proof. If $N$, respectively $\Gamma$, is of rank one, then condition (2) in Theorem 4.1 does not hold by Proposition 3.7. If $N$ is an irreducible locally symmetric space of non-compact type, then, by the definition of irreducibility, condition (3) does not hold. In all other cases, a result of Ballmann-Eberlein [3, Theorem $\mathrm{C}]$ shows that condition (3) does hold.

Theorem 4.1 has the following extension to rationally essential manifolds:

Corollary 4.4. Let $M$ be a closed, oriented, manifold whose fundamental group is also the fundamental group of a closed manifold $N$ admitting a Riemannian metric of non-positive sectional curvature. If $M$ is rationally essential and $P \geq M$ for a non-trivial product $P$ of closed manifolds, then $N$ has a finite covering $\bar{N}$ that is diffeomorphic to a non-trivial product.

Proof. Theorem 1.4 shows that $P \geq M$ implies statement (2) from Theorem 4.1 for the fundamental group $\pi_{1}(M)=\pi_{1}(N)$. The implication from (2) to (4) in Theorem 4.1 gives the conclusion.

Remark 4.5. In the case that every finite index subgroup of $\pi_{1}(N)$ has trivial centre, the combination of Theorems 1.4 and 4.1 shows that in the situation of Corollary 4.4 with $P=X_{1} \times X_{2} \geq M$, each $X_{i}$ is rationally essential in the corresponding factor of a finite covering of $N$.

To end this section, we point out that many non-positively curved Riemannian manifolds of rank one are comparable to direct products in the relation $\geq$. For example, closed hyperbolic Riemann surfaces $N$ satisfy $N \geq S^{1} \times S^{1}$. More interestingly, the branched covering construction of Fornari-Schroeder [12] produces rank one manifolds $N$ of higher dimension that are close to products in a certain geometric sense, and that by construction satisfy $N \geq\left(X_{1} \times X_{2}\right)$ for suitable $X_{i}$. 


\section{FibRe BUNDLES}

In this section we consider fibrations whose base and fibre have fundamental groups not presentable by products.

Theorem 5.1. Let $M$ be a closed, oriented, connected manifold that is the total space of a fibration whose base $B$ and fibre $F$ are closed, oriented, connected aspherical manifolds. Assume that $\pi_{1}(B)$ and $\pi_{1}(F)$ are not presentable by products. If $P \geq M$ for some non-trivial product $P=X_{1} \times X_{2}$ of closed, oriented, connected manifolds, then $M$ is finitely covered by a product whose factors are finite coverings of $B$ and $F$ respectively and are dominated by the $X_{i}$.

Remark 5.2. In the same way that Theorem 4.1 implies Corollary 4.4, Theorem 5.1 has consequences for rationally essential manifolds in place of aspherical ones.

Proof. Suppose there are closed, oriented, connected manifolds $X_{i}$ such that their product $P=X_{1} \times X_{2}$ admits a map $f: P \longrightarrow M$ of degree $d \neq 0$. Proposition 2.2 shows that the images $\Gamma_{i}$ of the fundamental groups of the factors $X_{i}$ are both infinite and together generate a finite index subgroup $\Gamma$ of $\pi_{1}(M)$. Applying Proposition 3.9 (and its proof) to $\Gamma$ we see that $\Gamma \cong \Gamma_{1} \times \Gamma_{2}$ and that $\Gamma_{1}$ and $\Gamma_{2}$ are finite index subgroups of $\pi_{1}(B)$ and $\pi_{1}(F)$ respectively. In particular, $M$ is finitely covered by a manifold $\bar{M}$ whose fundamental group is the direct product of the finite index subgroups $\Gamma_{i}$ of $\pi_{1}(B)$ and $\pi_{1}(F)$ respectively.

As the base $B$ and fibre $F$ are aspherical, so is $M$. It follows that $\bar{M}$ is homotopy equivalent to the product of finite covers of $B$ and $F$ corresponding to $\Gamma_{1}$ and $\Gamma_{2}$. Proposition 2.2 shows that these factors are dominated by the $X_{i}$.

The above proof shows that a finite covering of $M$ is homotopy equivalent to a trivial bundle. In specific situations one can sometimes prove more. In this direction we have for example:

Corollary 5.3. Let $M$ be a surface bundle over a surface with base $B$ and fibre $F$ both of genus $\geq 2$, and let $\Gamma:=\pi_{1}(M)$. The following are equivalent:

(1) $P \geq M$ for some non-trivial product $P$ of closed, oriented manifolds,

(2) the fundamental group $\Gamma$ is presentable by a product,

(3) a finite index subgroup of $\Gamma$ splits as a non-trivial direct product,

(4) there is a finite covering of $M$ diffeomorphic to a trivial surface bundle.

It is well known that the last condition is equivalent to the finiteness of the image of the monodromy representation of $\pi_{1}(B)$ in the mapping class group of $F$. 
Proof. As the base and fibre are negatively curved, their fundamental groups are not presentable by products by Proposition 3.6. The proof of Theorem 5.1 shows that the first three conditions are equivalent, and that they are equivalent to $M$ having a finite cover that is homotopy equivalent to trivial surface bundle. By a result of Hillman [19, Corollary 5.6.4 on p. 94] we may assume that this homotopy equivalence is a fibre-preserving diffeomorphism. (The assumption $\chi(B)<\chi(F)$ in Hillman's result can always be arranged by pulling back to a finite covering of the base.)

Corollary 5.3 is interesting from several different points of view. First of all, there are many examples of surface bundles over surfaces with both fibre and base of genus $\geq 2$ that do not admit any metric of non-positive sectional curvature [23]. Thus Corollary 5.3 cannot be deduced from the results of the previous section, and Theorem 5.1 is of a different nature than the differentialgeometric results of that section. Second of all, no surface bundle over a surface is known to admit a negatively curved metric, so that Corollary 5.3 has no known overlap with results about maps of products to negatively curved targets.

In higher dimensions, Theorem 5.1 and its potential generalisations from bundles to other aspherical manifolds raise the following question: If $M$ is a closed, oriented, connected, aspherical manifold whose fundamental group splits as a non-trivial direct product $\Gamma_{1} \times \Gamma_{2}$, can $M$ be split up to homotopy or homeomorphism as a product of closed, oriented, connected manifolds with fundamental group $\Gamma_{1}$ and $\Gamma_{2}$ respectively? Using the sophisticated machinery developed in the field of topological rigidity, this question can be answered affirmatively for a large class of such manifolds [28]. This solution relies on deep results concerning the Farrell-Jones conjecture, the Borel conjecture, the Novikov conjecture, and the resolution of homology manifolds. For non-positively curved manifolds, we have seen in the previous section that the geometric arguments in the proof of the Gromoll-Wolf splitting theorem [13] take care of these complications.

\section{Amenable Centralisers}

In our discussion so far we have typically assumed that the target manifolds are rationally essential, and that their fundamental groups have small centralisers. For the groups occurring in Theorem 1.5, the centralisers are as small as possible, that is (virtually) cyclic. This bound on the size of centralisers was not needed for all elements, but for the generic ones, e.g., the elements of infinite order in hyperbolic groups, or the pseudo-Anosov elements in mapping class groups. 
In this section we treat groups with larger centralisers, which are not necessarily virtually Abelian. The price to be paid is that rational essentialness has to be replaced by a stronger assumption. As an example of such a generalisation we consider fundamental groups with amenable centralisers and manifolds with non-zero simplicial volume. For background on the simplicial volume we refer to the work of Gromov [14] and the exposition by Ivanov [20], and for information on amenability to Paterson's book [30].

Theorem 6.1. Let $M$ be a closed, oriented, connected manifold with non-zero simplicial volume. Assume that $\pi_{1}(M)$ contains an element of infinite order, and that every element of infinite order has amenable centraliser. Then $P \ngtr M$ for any non-trivial product $P$ of closed, oriented, connected manifolds.

Proof. Suppose for a contradiction that $P=X_{1} \times X_{2}$ is a non-trivial product of closed, oriented, connected manifolds admitting a map $f: P \longrightarrow M$ of degree $d \neq 0$. By Lemma 2.1 we have commuting subgroups $\Gamma_{1}, \Gamma_{2} \subset \pi_{1}(M)$ such that the multiplication map $\Gamma_{1} \times \Gamma_{2} \longrightarrow \pi_{1}(M)$ is surjective onto a subgroup $\Gamma \subset \pi_{1}(M)$ of finite index.

The assumption that $\pi_{1}(M)$ contains an element of infinite order implies that the same is true for one of the $\Gamma_{i}$. If $g_{1} \in \Gamma_{1}$ has infinite order, then by the assumption about centralisers, the centraliser $C_{\Gamma}\left(g_{1}\right)$ of $g_{1}$ in $\Gamma$, which is a subgroup of the amenable group $C_{\pi_{1}(M)}\left(g_{1}\right)$, is amenable. But $\Gamma_{2} \subset C_{\Gamma}\left(g_{1}\right)$, because the $\Gamma_{i}$ commute. Therefore $\Gamma_{2}$ is amenable.

Proposition 2.2 also holds for homology with real coefficients, and so we have homology classes $\alpha_{1} \in H_{\operatorname{dim} X_{1}}\left(B \Gamma_{1} ; \mathbb{R}\right)$ and $\alpha_{2} \in H_{\operatorname{dim} X_{2}}\left(B \Gamma_{2} ; \mathbb{R}\right)$ satisfying

$$
H_{n}\left(c_{M}\right)(d \cdot[M])=H_{n}(\overline{B \varphi})\left(\alpha_{1} \times \alpha_{2}\right) .
$$

We now apply the functorial $\ell^{1}$-semi-norm to this equation. On the one hand, by the assumption on the simplicial volume of $M$ and the degree $d$, the mapping theorem for bounded cohomology [14, p. 40/17] yields

$$
\left\|H_{n}\left(c_{M}\right)(d \cdot[M])\right\|_{1}=|d| \cdot\|M\| \neq 0 .
$$

On the other hand, $\left\|\alpha_{2}\right\|_{1}=0$ because $\Gamma_{2}$ is amenable [14, p. 40/17], [20, Theorem 4.3], which implies

$$
\left\|H_{n}(\overline{B \varphi})\left(\alpha_{1} \times \alpha_{2}\right)\right\|_{1} \leq 2^{\operatorname{dim} M} \cdot\left\|\alpha_{1}\right\|_{1} \cdot\left\|\alpha_{2}\right\|_{1}=0
$$

by the almost-multiplicativity of the norm. This contradiction completes the proof that $P \nsupseteq M$.

The assumption that all elements of infinite order have amenable centralisers is quite restrictive, but can in practice be relaxed. For example, we have the following variation on Theorem 6.1: 
Corollary 6.2. Let $M$ be a closed, oriented, connected manifold with non-zero simplicial volume. Assume that $\pi_{1}(M)$ fits into an extension

$$
1 \longrightarrow K \longrightarrow \pi_{1}(M) \stackrel{\pi}{\longrightarrow} Q \longrightarrow 1
$$

where $K$ is amenable and $Q$ is a torsion-free group in which every non-trivial element has an amenable centraliser. Then $P \nsupseteq M$ for any non-trivial product $P$ of closed, oriented, connected manifolds.

Proof. If $f: P \longrightarrow M$ is of non-zero degree, we can again apply Lemma 2.1 to find commuting subgroups $\Gamma_{1}, \Gamma_{2} \subset \pi_{1}(M)$ for which the multiplication map $\Gamma_{1} \times \Gamma_{2} \longrightarrow \pi_{1}(M)$ is surjective onto a subgroup of $\pi_{1}(M)$ of finite index. It follows that $\pi\left(\Gamma_{1}\right)$ and $\pi\left(\Gamma_{2}\right)$ are commuting subgroups of $Q$ generating a finite index subgroup. If $\pi\left(\Gamma_{1}\right)$ is non-trivial, then it follows from the assumption about centralisers in $Q$ that $\pi\left(\Gamma_{2}\right)$ is amenable. As the class of amenable groups is closed under extensions, we conclude that $\Gamma_{2}$ is amenable, which gives a contradiction as in the proof of Theorem 6.1. If $\pi\left(\Gamma_{1}\right)$ is trivial, then $\Gamma_{1} \subset K$ is amenable because it is a subgroup of an amenable group, and we again have a contradiction.

Corollary 6.2 excludes domination by products for many manifolds whose fundamental groups are presentable by products. For example, the extension (3) could be central, so that $K$ is Abelian and in the centre of $\pi_{1}(M)$. In this situation Lemma 3.1 implies that $\pi_{1}(M)$ is presentable by a product, so that Theorem 1.4 cannot be used to prove $P \nsupseteq M$. In general, subexponential extensions in the sense of Sambusetti [33] provide examples of amenable extensions, because any group of subexponential growth is amenable.

Here is a concrete example demonstrating the applicability of Corollary 6.2.

Example 6.3. Let $X=\mathbb{C} H^{2} / Q$ be a smooth compact complex ball quotient. Then $X$ is a complex-algebraic surface whose fundamental group $Q$ is torsion-free and hyperbolic (and therefore has virtually cyclic centralisers). In particular it satisfies the assumptions made on $Q$ in Corollary 6.2. Let $Y$ be an elliptic curve, and $M \subset X \times Y$ a smooth hyperplane section. Then $\pi_{1}(M)=\pi_{1}(X \times Y)=Q \times \mathbb{Z}^{2}$ by the Lefschetz hyperplane theorem. Moreover, the projection of $X \times Y$ to the first factor restricts to a surjective holomorphic map $M \longrightarrow X$, which shows $M \geq X$. As $X$ has strictly negative sectional curvature, its simplicial volume is positive, and the relation $M \geq X$ shows that $M$ also has positive simplicial volume. Thus Corollary 6.2 shows $P \nsupseteq M$ for any non-trivial product $P$, although $\pi_{1}(M)$ is a product. 
On the one hand, this example certainly shows that Corollary 6.2 is not empty, although it is conjectured that a closed aspherical manifold whose fundamental group contains a non-trivial amenable normal subgroup has vanishing simplicial volume. The manifold $M$ in the example is rationally essential with non-zero simplicial volume, but not aspherical. On the other hand, for this particular $M$ the conclusion $P \nsucceq M$ also follows from the combination of $M \geq X$ and $P \nsupseteq X$, with the latter being a consequence of Theorems 1.4 and 1.5 .

\section{OUTLOOK}

In this section we discuss the relation between the domination relation and the ranks of fundamental groups, and we also consider the domination relation in general, without restricting to product domains and to targets with large fundamental groups.

Relationship with the rank of groups. Building on ideas of Tits, Prasad and Raghunathan, Ballmann and Eberlein [3] introduced an abstract notion of the rank of a group $\Gamma$. This notion is a measure of the size of centralisers of generic elements for all finite index subgroups of $\Gamma$. In particular, the rank does not change under passage to a subgroup of finite index. It is a result of Prasad-Raghunathan [31] that the rank of a cocompact lattice in the isometry group of a symmetric space of non-compact type coincides with the rank (in the sense of Lie theory) of the symmetric space. Ballmann-Eberlein [3] proved that for the fundamental groups of closed manifolds of non-positive sectional curvature the rank agrees with the geometric rank of the Riemannian metric defined via spaces of parallel Jacobi fields (which in turn agrees with the Lie theoretic rank in the case of locally symmetric spaces).

The groups occurring in Theorem 1.5 are all of rank one. In the statement (N-P) this is part of the assumption because, by the result of BallmannEberlein, the geometric and algebraic rank are the same. For infinite hyperbolic groups it is well-known folklore that they have rank one - indeed one could argue that this is part of the definition, or at least of the philosophy behind their definition [15]. For mapping class groups the determination of the rank is a theorem of Ivanov [21].

It is possible that Theorem 1.5 is a special case of a more general result asserting that rank one groups in the sense of Ballmann-Eberlein that are not virtually cyclic are not presentable by products. The proof of such a statement in the general case runs into many algebraic technicalities, so we do not pursue it here. Moreover, in concrete cases it has turned out to be easier to prove directly that a group is not presentable by a product, instead of proving that 
it has rank one. A case in point is provided by the mapping class groups, for which our proof of statement (MCG) in Theorem 1.5 is easier than Ivanov's proof $[21]$ that the groups have rank one.

The domination relation in general. In this paper we have shown that many manifolds with large fundamental groups are not dominated by products. In contrast with this, it could be true that all manifolds with finite fundamental groups are dominated by products. Using the results of Duan-Wang [8, 9] this is easy to verify in dimension four:

Proposition 7.1. Every closed oriented connected four-manifold with finite fundamental group is dominated by the product of a torus with a suitable closed oriented connected surface.

Proof. Clearly it is enough to prove the statement for simply connected targets.

Let $P$ and $N$ be closed oriented four-manifolds with intersection forms $Q_{P}$ and $Q_{N}$ respectively. If $f: P \longrightarrow N$ is a map of degree $d$, then $d \cdot Q_{N}$ is embedded into $Q_{P}$ by the pullback $H^{*}(f)$. (Here $d \cdot Q_{N}$ means that all entries are multiplied by $d$.) It is a result of Duan and Wang [9, Theorem 3] that for simply connected targets $N$ a map of degree $d$ exists whenever the necessary algebraic condition $d \cdot Q_{N} \subset Q_{P}$ is satisfied.

We apply this in the case where $P=S^{1} \times S^{1} \times \Sigma_{g}$ for some $g \in \mathbb{N}$. This $P$ has zero signature, so that the embedding of intersection forms exists whenever the second Betti number of $P$ is large enough compared with the positive and negative parts of $Q_{N}$ (which may have different rank because $N$ may have non-zero signature). If $Q_{N}$ is odd, we have to choose $d$ even to find such an algebraic embedding, because $Q_{P}$ is even. It suffices to take $g$ large with respect to $b_{2}^{ \pm}(N)$.

Carlson and Toledo [7, p. 174] mentioned that in arbitrary dimensions there is no easily described class of manifolds forming maximal elements for the relation $\geq$ in the sense that for every closed oriented $M$ there should be an $N$ from this class such that $N \geq M$. In this direction we propose the following:

Conjecture 7.2. In every dimension $n \geq 2$, closed oriented hyperbolic manifolds represent a maximal class of homotopy types with respect to the domination relation $\geq$.

This is trivially true for $n=2$, and, more interestingly, it is known to be true for $n=3$ by a result of Brooks [5]. We can also verify a strong form of the conjecture for four-manifolds with finite fundamental groups: 
Proposition 7.3. Let $M$ be a closed oriented connected four-manifold with finite fundamental group. Then every closed oriented hyperbolic manifold $N$ virtually dominates $M$, i.e., some finite covering space of $N$ dominates $M$.

Proof. The proof is almost the same as the proof of Proposition 7.1. Like that proof, this one is a direct consequence of the result of Duan-Wang [9, Theorem 3], because we may assume $M$ to be simply connected.

Fix a simply connected closed oriented four-manifold $M$ and a closed oriented hyperbolic four-manifold $N$. We want to show that $N$ virtually dominates $M$. Recall that $N$ has positive Euler characteristic and zero signature [26]. Moreover, $\pi_{1}(N)$ is a residually finite lattice, and so $N$ has finite coverings $\bar{N}$ of arbitrarily large degrees, equivalently of arbitrarily large Euler characteristics. This means that the second Betti numbers of these coverings are unbounded, and once they are large enough, we can verify the algebraic criterion $d \cdot Q_{M} \subset Q_{\bar{N}}$ of Duan-Wang [9, Theorem 3], say with $d=2$.

Finally, in support of Conjecture 7.2, we would like to mention the following heuristic. A candidate class of maximal elements with respect to $\geq$ should consist of manifolds that are not themselves dominated by too many other manifolds, because otherwise the transitivity of the relation might lead to contradictions. Hyperbolic manifolds are indeed dominated by very few other manifolds: not by non-trivial products (by our results), not by manifolds with zero simplicial volume [14], not by Kähler manifolds [7, 1], and not even by certain Kähler-Weyl manifolds [25].

\section{REFERENCES}

1. J. Amorós, M. Burger, K. Corlette, D. Kotschick and D. Toledo, Fundamental Groups of Compact Kähler Manifolds, Mathematical Surveys and Monographs, Vol. 44, Amer. Math. Soc., Providence, R.I. 1996.

2. W. Ballmann, M. Brin and P. Eberlein, Structure of manifolds of nonpositive curvature, Ann. of Math. 122 (1985), 171-203.

3. W. Ballmann and P. Eberlein, Fundamental groups of manifolds of nonpositive curvature, J. Differential Geometry 25 (1987), 1-22.

4. M. R. Bridson and A. Haefliger, Metric Spaces of Non-positive Curvature, Grundlehren der Mathematischen Wissenschaften Vol. 319, Springer Verlag 1999.

5. R. Brooks, On branched coverings of 3-manifolds which fiber over the circle, J. Reine Angew. Math. 362 (1985), 87-101.

6. M. Brunnbauer, Homological invariance for asymptotic invariants and systolic inequalities, Geom. Funct. Analysis DOI 10.1007/s00039-008-0677-4 (online first, to appear in print).

7. J. A. Carlson and D. Toledo, Harmonic mapping of Kähler manifolds to locally symmetric spaces, Publ. Math. I.H.E.S. 69 (1989), 173-201. 
8. H. Duan and S. Wang, The degrees of maps between manifolds, Math. Z. 244 (2003), 67-89.

9. H. Duan and S. Wang, Non-zero degree maps between 2n-manifolds, Acta Math. Sin. (Engl. Ser.) 20 (2004), 1-14.

10. P. Eberlein, A canonical form for compact nonpositively curved manifolds whose fundamental groups have nontrivial center, Math. Annalen 260 (1982), 23-29.

11. P. Eberlein, Euclidean de Rham factor of a lattice of nonpositive curvature, J. Differential Geometry 18 (1983), 209-220.

12. S. Fornari and V. Schroeder, Ramified coverings with nonpositive curvature, Math. Z. 203 (1990), 123-128.

13. D. Gromoll and J. A. Wolf, Some relations between the metric structure and the algebraic structure of the fundamental group in manifolds of nonpositive curvature, Bull. Amer. Math. Soc. 77 (1971), 545-552.

14. M. Gromov, Volume and bounded cohomology, Publ. Math. I.H.E.S. 56 (1982), 5-99.

15. M. Gromov, Hyperbolic groups, in Essays in Group Theory (ed. S. M. Gersten), MSRI Publ. 8, Spinger Verlag 1987, pp. 75-263.

16. M. Gromov, Metric Structures for Riemannian and Non-Riemannian Spaces, with appendices by M. Katz, P. Pansu and S. Semmes, translated from the French by S. M. Bates, Progress in Mathematics Vol. 152, Birkhäuser Verlag 1999.

17. B. Hanke, D. Kotschick, J. Roe and T. Schick, Coarse topology, enlargeability and essentialness, Ann. Sci. Ecole Norm. Sup. 41 (2008), 471-493.

18. B. Hanke and T. Schick, Enlargeability and index theory, J. Differential Geom. 74 (2006), 293-320.

19. J. A. Hillman, Four-manifolds, geometries and knots, Geometry and Topology Monographs Vol. 5 (2002).

20. N. V. Ivanov, Foundations of the theory of bounded cohomology, J. Soviet Math. 37 (1987), 1090-1114.

21. N. V. Ivanov, Rank of Teichmüller modular groups, (Russian) Mat. Zametki 44 (1988), 636-644; English translation in Math. Notes 44 (1988), 829-832.

22. N. V. Ivanov, Subgroups of Teichmüller Modular Groups, Translations of Math. Monographs, Vol. 115, American Math. Soc., Providence, R.I. 1992.

23. M. Kapovich and B. Leeb, Actions of discrete groups on nonpositively curved spaces, Math. Annalen 306 (1996), 341-352.

24. J. Kędra, D. Kotschick and S. Morita, Crossed flux homomorphisms and vanishing theorems for flux groups, Geom. Funct. Analysis 16 (2006), 1246-1273.

25. G. Kokarev and D. Kotschick, Fibrations and fundamental groups of Kähler-Weyl manifolds, Preprint arXiv:0811.1952 v1 [math.DG] 12 Nov 2008.

26. D. Kotschick, Remarks on geometric structures on compact complex surfaces, Topology 31 (1992), 317-321.

27. H. B. Lawson, Jr. and S.-T. Yau, Compact manifolds of nonpositive curvature, J. Differential Geom. 7 (1972), 211-228.

28. W. Lück, Survey on aspherical manifolds, preliminary version, 2008.

29. J. W. Milnor and W. P. Thurston, Characteristic numbers of 3-manifolds, Enseign. Math. 23 (1977), 249-254; reprinted in J. W. Milnor, Collected Papers, Vol. 2, Publish or Perish 1995. 
30. A. L. T. Paterson, Amenability, Mathematical Surveys and Monographs, Vol. 29, Amer. Math. Soc., Providence, R.I. 1988.

31. G. Prasad and M. S. Raghunathan, Cartan subgroups and lattices in semisimple groups, Ann. of Math. 96 (1972), 296-317.

32. M. S. Raghunathan, Discrete Subgroups of Lie Groups, Vol. 68 of Ergebnisse der Mathematik und ihrer Grenzgebiete, Springer Verlag 1972.

33. A. Sambusetti, Minimal entropy and simplicial volume, Manuscripta math. 99 (1999), 541-560.

34. S. Wang, Non-zero degree maps between 3-manifolds, Proc. of the ICM Beijing 2002 Vol. II 457-468, Higher Education Press Beijing 2002.

35. D. Witte Morris, Introduction to Arithmetic Groups. Preliminary book, 2001. Available at arXiv:math/0106063v3 [math.DG]

Mathematisches Institut, LMu München, Theresienstr. 39, 80333 München, Germany

E-mail address: dieter@member.ams.org

Mathematisches Institut, WWU Münster, Einsteinstr. 62, 48143 Münster, Germany

E-mail address: clara.loeh@uni-muenster.de 